\title{
Erratum to: Characterisation of the protein corona using tunable resistive pulse sensing: determining the change and distribution of a particle's surface charge
}

\author{
Emma L. C. J. Blundell ${ }^{1}$ - Matthew J. Healey ${ }^{1} \cdot$ Elizabeth Holton $^{1}$ • \\ Muttuswamy Sivakumaran $^{2} \cdot$ Sarabjit Mastana $^{3} \cdot$ Mark Platt $^{1}$
}

Received: 4 October 2016 / Accepted: 4 October 2016 / Published online: 15 October 2016

(C) Springer-Verlag Berlin Heidelberg 2016

Erratum to: Anal Bioanal Chem

DOI: 10.1007/s00216-016-9678-6

The authors would like to call the reader's attention to the fact that unfortunately Sarabjit Mastana's family name was misspelled in the published article. The correct family name is Mastana.

The online version of the original article can be found at http://dx.doi. org/10.1007/s00216-016-9678-6.

Mark Platt

m.platt@lboro.ac.uk

1 Department of Chemistry, Loughborough University, Loughborough LE11 3TU, UK

2 Peterborough City Hospital, Edith Cavell Campus, Bretton Gate, Peterborough PE3 9GZ, UK

3 Human Genomics Lab, Centre for Global Health and Human Development, School of Sport, Exercise and Health Sciences, Loughborough University, Loughborough, UK 